\title{
Correction to: Textural, Color, Hygroscopic, Lipid Oxidation, and Sensory Properties of Cookies Containing Free and Microencapsulated Chia Oil
}

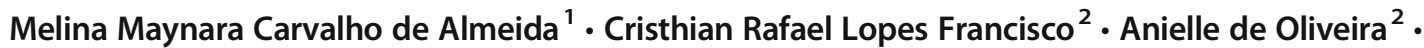
Sabrina Silva de Campos ${ }^{2}$ - Ana Paula Bilck ${ }^{3}$ - Renata Hernandez Barros Fuchs ${ }^{2}$ • Odinei Hess Gonçalves ${ }^{1}$. Patrícia Valderrama ${ }^{1}$. Aziza Kamal Genena ${ }^{1}$. Fernanda Vitória Leimann ${ }^{1}$

Published online: 19 February 2018

(C) Springer Science+Business Media, LLC, part of Springer Nature 2018

\section{Correction to: Food and Bioprocess Technology} https://doi.org/10.1007/s11947-018-2057-x

The original version of this article unfortunately a typo error on the name of Dr. Patrícia Valderrama. This was written as "Patrícia Velderrama" which is incorrect.

Herewith, the authors corrected name as given above.

The online version of the original article can be found at https://oi.org/ 10.1007/s11947-018-2057-x

Fernanda Vitória Leimann

fernandaleimann@utfpr.edu.br

1 Programa de Pós-Graduação em Tecnologia de Alimentos (PPGTA), Universidade Tecnológica Federal do Paraná, Campus Campo Mourão (UTFPR-CM), via RosalinaMaria Dos Santos, 1233, Caixa, Postal: 271, Campo Mourão, Paraná CEP 87301-899, Brazil

2 Departamento Acadêmico de Alimentos (DALIM), Universidade Tecnológica Federal do Paraná, Campus Campo Mourão (UTFPR-CM), via Rosalina Maria Dos Santos, 1233, Caixa Postal: 271, Campo Mourão, Paraná CEP 87301-899, Brazil

3 Departamento de Ciência e Tecnologia de Alimentos, Centro de Ciências Agrárias, Universidade Estadual de Londrina (UEL), Rod. Celso Garcia Cid (PR 445), Km 380, Caixa Postal 10.011,

Londrina, PR CEP: 86057-970, Brazil 